\title{
7 Production, output and costs
}

\subsection{Oupput of the production industries}

Average $2003=100$

\begin{tabular}{|c|c|c|c|c|c|c|c|c|c|c|c|}
\hline & \multicolumn{11}{|c|}{ Summary - Seasonally adjusted } \\
\hline & \multirow[b]{2}{*}{$\begin{array}{r}\text { Total } \\
\text { production } \\
\text { industries }\end{array}$} & \multirow[b]{2}{*}{$\begin{array}{l}\text { Mining and } \\
\text { quarrying }\end{array}$} & \multicolumn{8}{|c|}{ Manufacturing industries } & \multirow[b]{2}{*}{$\begin{array}{r}\text { Electricity, } \\
\text { gas and } \\
\text { water }\end{array}$} \\
\hline & & & $\begin{array}{r}\text { Total } \\
\text { manufact- } \\
\text { uring } \\
\text { industries }\end{array}$ & $\begin{array}{l}\text { Food, drink } \\
\text { and tobacco }\end{array}$ & $\begin{array}{r}\text { Textiles, } \\
\text { leather and } \\
\text { clothing }\end{array}$ & $\begin{array}{r}\text { Coke ref } \\
\text { petrol and } \\
\text { nuclear } \\
\text { fuels }\end{array}$ & $\begin{array}{r}\text { Chemicals } \\
\text { and man-made } \\
\text { fibres }\end{array}$ & $\begin{array}{c}\text { Basic metals } \\
\text { and metal } \\
\text { products }\end{array}$ & $\begin{array}{c}\text { Engineering } \\
\text { and allied } \\
\text { industries }\end{array}$ & $\begin{array}{r}\text { Other } \\
\text { manufact- } \\
\text { uring }\end{array}$ & \\
\hline \multicolumn{12}{|l|}{ SIC 2003} \\
\hline Weights & 1000.0 & 117.4 & 792.8 & 117.2 & 26.0 & 13.0 & 88.4 & 80.8 & 238.6 & 228.8 & 89.8 \\
\hline & CKYW & CKYX & CKYY & CKZA & AGVO & CKZF & CKZG & CKZJ & AGXS & $A G X Q$ & CKYZ \\
\hline 2003 & 100.0 & 100.0 & 100.0 & 100.0 & 100.0 & 100.0 & 100.0 & 100.0 & 100.0 & 100.0 & 100.0 \\
\hline 2004 & 100.9 & 92.1 & 102.2 & 102.0 & 89.5 & 107.8 & 103.6 & 102.7 & 104.4 & 100.4 & 101.0 \\
\hline 2005 & 99.8 & 84.2 & 102.0 & 103.6 & 87.8 & 111.0 & 106.5 & 103.6 & 103.8 & 98.1 & 100.7 \\
\hline 2006 & 100.5 & 78.0 & 103.8 & 102.9 & 88.0 & 105.4 & 110.0 & 105.1 & 108.0 & 98.9 & 100.1 \\
\hline 2007 & 100.9 & 76.7 & 104.4 & 102.3 & 86.4 & 109.3 & 108.8 & 106.4 & 109.7 & 99.3 & 101.2 \\
\hline \multicolumn{12}{|c|}{ Seasonally adjusted } \\
\hline 2004 Q2 & 101.4 & 92.9 & 102.6 & 102.2 & 89.5 & 105.5 & 104.2 & 102.4 & 104.7 & 101.4 & 101.6 \\
\hline Q3 & 100.3 & 92.5 & 101.4 & 101.8 & 87.1 & 104.5 & 101.7 & 102.7 & 103.9 & 99.3 & 100.6 \\
\hline Q4 & 100.6 & 89.4 & 102.4 & 102.5 & 86.6 & 110.3 & 104.4 & 104.5 & 105.0 & 99.5 & 99.4 \\
\hline 2005 Q1 & 100.2 & 87.0 & 102.1 & 103.9 & 88.6 & 123.3 & 104.7 & 102.3 & 103.1 & 99.3 & 100.7 \\
\hline Q2 & 100.4 & 86.2 & 102.4 & 103.8 & 89.0 & 110.3 & 106.3 & 103.7 & 104.6 & 98.3 & 101.3 \\
\hline Q3 & 99.7 & 82.2 & 102.2 & 103.8 & 87.1 & 107.6 & 107.4 & 103.2 & 104.7 & 97.7 & 100.2 \\
\hline Q4 & 99.0 & 81.6 & 101.4 & 103.1 & 86.4 & 102.5 & 107.7 & 105.0 & 102.6 & 97.1 & 100.6 \\
\hline 2006 Q1 & 100.6 & 82.6 & 102.9 & 102.0 & 89.4 & 105.1 & 107.9 & 104.1 & 106.2 & 99.0 & 103.7 \\
\hline Q2 & 100.4 & 77.3 & 103.8 & 102.6 & 87.8 & 106.7 & 110.0 & 105.2 & 108.3 & 98.6 & 99.7 \\
\hline Q3 & 100.6 & 77.1 & 104.3 & 102.9 & 86.6 & 104.3 & 111.4 & 105.8 & 108.5 & 99.4 & 99.3 \\
\hline Q4 & 100.3 & 75.1 & 104.3 & 104.0 & 88.2 & 105.3 & 110.8 & 105.3 & 109.0 & 98.6 & 97.9 \\
\hline 2007 Q1 & 100.2 & 76.5 & 103.8 & 102.7 & 84.6 & 108.0 & 109.4 & 106.1 & 108.7 & 98.1 & 99.6 \\
\hline Q2 & 101.0 & 77.4 & 104.7 & 102.8 & 86.8 & 106.9 & 108.8 & 106.8 & 110.2 & 99.5 & 99.7 \\
\hline 2008 Q1 & $100.6^{\dagger}$ & $72.6^{\dagger}$ & $104.6^{\dagger}$ & 102.3 & $87.0^{\dagger}$ & $115.9^{\dagger}$ & $111.0^{\dagger}$ & $104.5^{\dagger}$ & $109.7^{\dagger}$ & $99.2^{\dagger}$ & $102.1^{\dagger}$ \\
\hline Q2 & 99.6 & 72.5 & 103.4 & 100.2 & 87.2 & 115.2 & 110.3 & 104.3 & 109.0 & 97.3 & 101.9 \\
\hline Q3 & 97.9 & 71.8 & 101.4 & $99.8^{\dagger}$ & 87.3 & 111.8 & 110.5 & 102.7 & 105.9 & 94.7 & 101.3 \\
\hline Q4 & 93.5 & 69.5 & 96.2 & 98.1 & 85.3 & 116.6 & 104.7 & 93.1 & 100.3 & 88.9 & 101.2 \\
\hline 2007 Jan & 100.5 & 75.7 & 104.1 & 103.5 & 84.2 & 111.9 & 110.4 & 106.3 & 109.1 & 97.9 & 101.0 \\
\hline Feb & 99.8 & 77.6 & 103.2 & 102.1 & 83.7 & 107.7 & 108.6 & 106.2 & 107.9 & 97.8 & 98.6 \\
\hline Mar & 100.3 & 76.4 & 103.9 & 102.4 & 85.9 & 104.6 & 109.3 & 105.8 & 109.2 & 98.5 & 99.2 \\
\hline Apr & 100.4 & 76.9 & 104.4 & 103.3 & 85.2 & 107.2 & 109.4 & 106.4 & 109.5 & 99.2 & 95.1 \\
\hline May & 101.4 & 78.3 & 104.8 & 102.8 & 87.4 & 108.0 & 108.8 & 106.7 & 110.1 & 99.8 & 101.5 \\
\hline Jun & 101.4 & 77.0 & 104.8 & 102.2 & 87.6 & 105.4 & 108.3 & 107.2 & 111.1 & 99.4 & 102.6 \\
\hline Jul & 100.9 & 77.6 & 104.4 & 102.2 & 87.0 & 106.7 & 107.5 & 106.8 & 110.1 & 99.3 & 100.9 \\
\hline Aug & 101.0 & 77.1 & 104.7 & 102.2 & 88.3 & 110.5 & 108.2 & 106.6 & 109.8 & 100.1 & 100.2 \\
\hline Sep & 100.9 & 75.3 & 104.5 & 102.0 & 87.5 & 112.6 & 108.2 & 106.6 & 109.8 & 99.4 & 103.2 \\
\hline Oct & 101.7 & 77.4 & 105.0 & 101.5 & 87.4 & 114.0 & 108.6 & 106.7 & 110.3 & 100.9 & 104.2 \\
\hline Nov & 101.2 & 75.3 & 104.7 & 102.1 & 85.9 & 111.5 & 109.0 & 105.9 & 109.6 & 100.5 & 104.1 \\
\hline Dec & 101.1 & 76.2 & 104.5 & 101.6 & 86.2 & 111.0 & 109.5 & 105.0 & 110.4 & 99.4 & 104.0 \\
\hline 2008 Jan & $100.7^{\dagger}$ & $72.9^{\dagger}$ & $104.7^{\dagger}$ & 102.3 & 86.5 & $114.6^{\dagger}$ & $110.7^{\dagger}$ & $103.8^{\dagger}$ & $110.1^{\dagger}$ & $99.7^{\dagger}$ & $101.9^{\dagger}$ \\
\hline Feb & 100.7 & 72.4 & 104.8 & 102.5 & $87.1^{\dagger}$ & 116.2 & 112.1 & 105.1 & 109.5 & 99.4 & 101.9 \\
\hline Mar & 100.4 & 72.4 & 104.2 & 102.1 & 87.3 & 116.9 & 110.3 & 104.6 & 109.4 & 98.6 & 102.5 \\
\hline Apr & 100.4 & 72.3 & 104.1 & $101.0^{\dagger}$ & 87.5 & 117.3 & 109.5 & 104.5 & 110.2 & 98.2 & 104.3 \\
\hline May & 99.4 & 72.6 & 103.4 & 100.4 & 87.2 & 112.6 & 110.4 & 103.9 & 109.1 & 97.4 & 99.6 \\
\hline Jun & 99.1 & 72.6 & 102.7 & 99.3 & 87.1 & 115.7 & 111.0 & 104.3 & 107.8 & 96.3 & 101.8 \\
\hline Jul & 98.6 & 70.5 & 102.3 & 99.3 & 88.2 & 111.7 & 111.6 & 103.6 & 107.1 & 95.7 & 102.6 \\
\hline Aug & 97.8 & 70.0 & 101.5 & 100.1 & 87.4 & 110.5 & 111.3 & 102.8 & 105.8 & 94.6 & 101.3 \\
\hline Sep & 97.4 & 74.8 & 100.5 & 100.0 & 86.3 & 113.2 & 108.4 & 101.8 & 104.7 & 93.6 & 100.0 \\
\hline Oct & 95.7 & 68.8 & 98.9 & 99.6 & 85.1 & 113.9 & 109.9 & 97.2 & 103.6 & 90.8 & 101.9 \\
\hline Nov & 93.3 & 70.0 & 95.9 & 98.5 & 86.4 & 115.6 & 103.0 & 93.0 & 99.6 & 89.0 & 100.4 \\
\hline Dec & 91.7 & 69.7 & 93.8 & 96.2 & 84.5 & 120.2 & 101.2 & 89.0 & 97.7 & 86.9 & 101.3 \\
\hline
\end{tabular}




\subsection{Output to the productionon industries

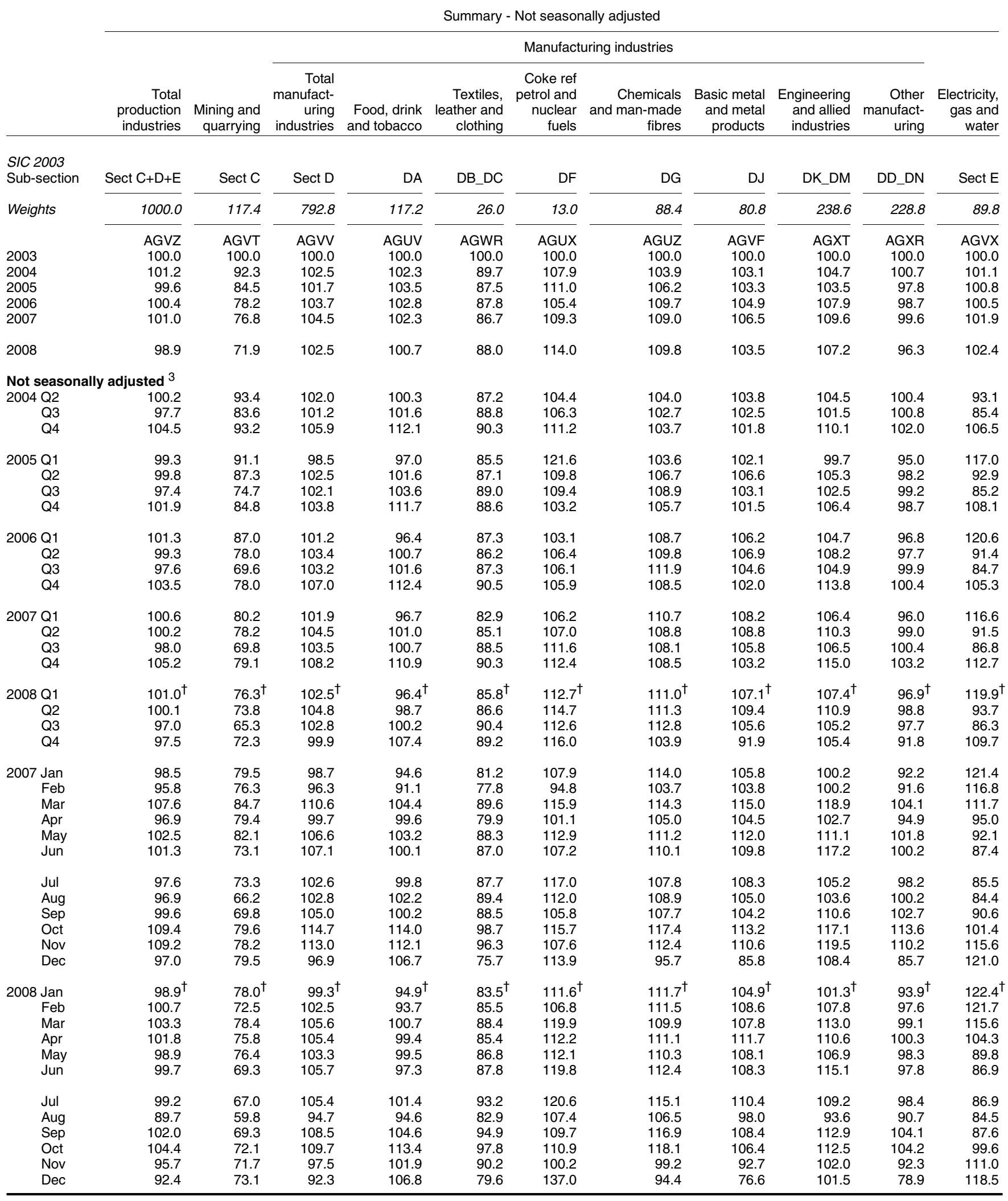


Production, output and costs

\subsection{Ouput of the production industries}

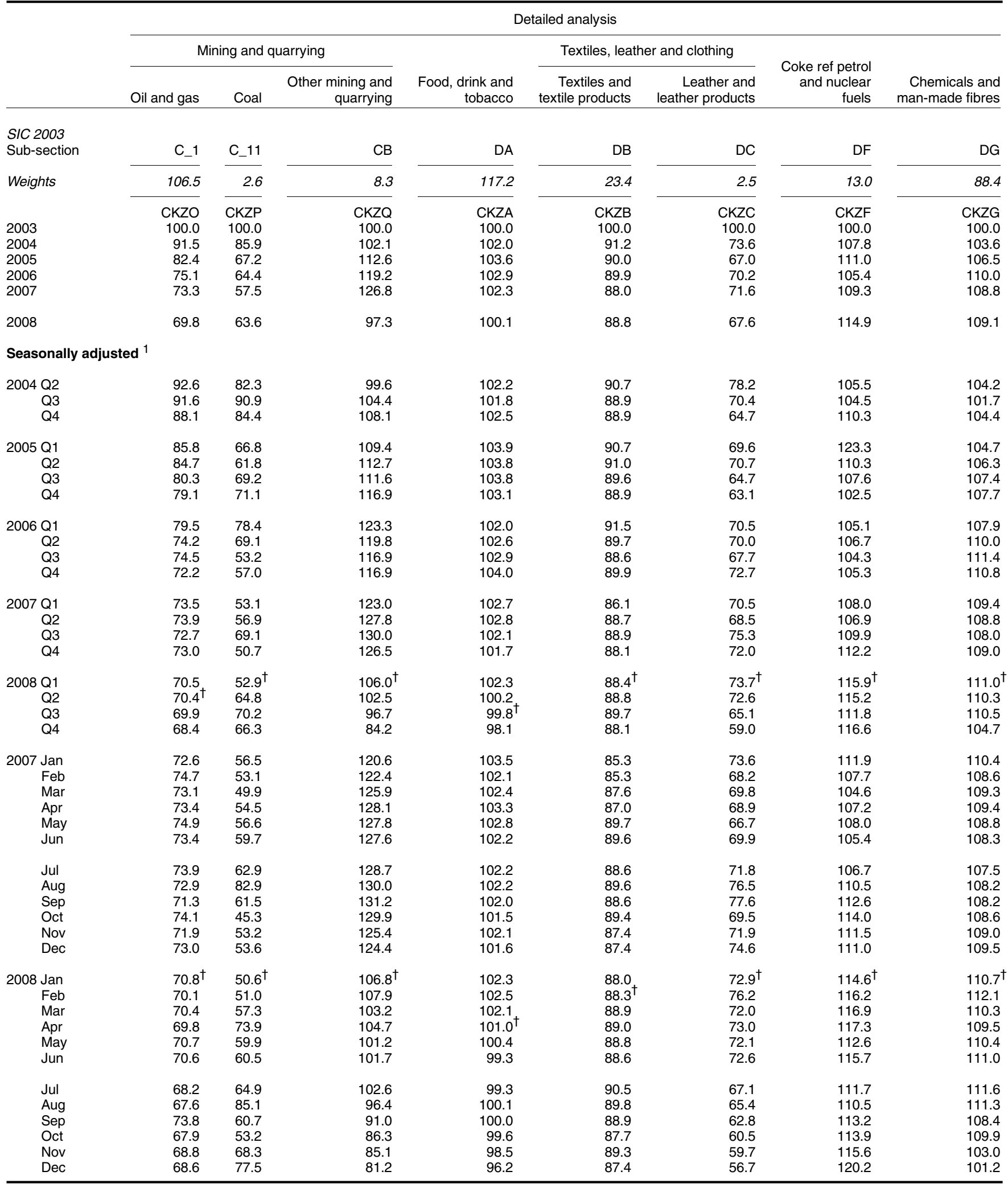




\subsection{Ouput of the production nindustries

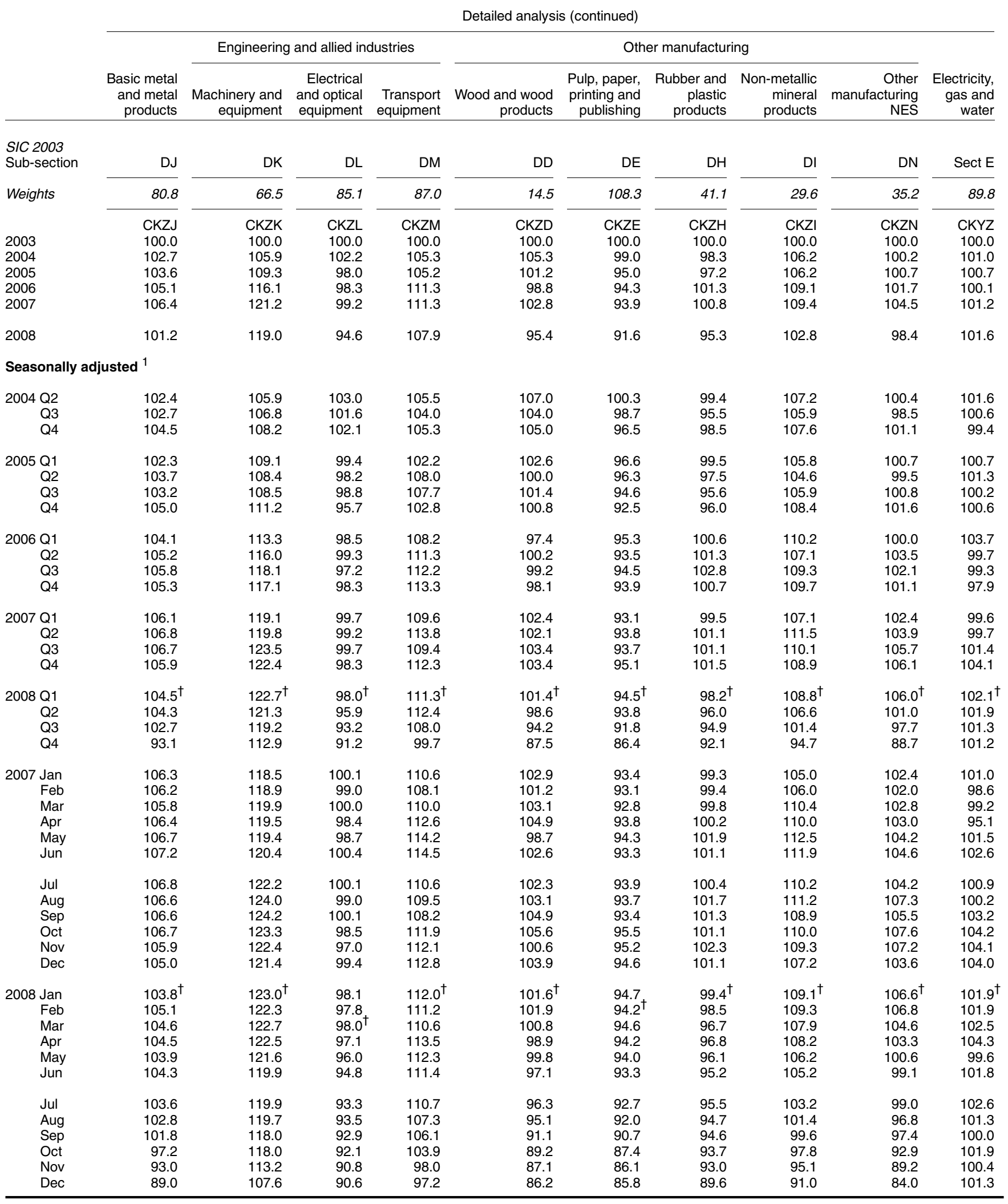


Production, output and costs

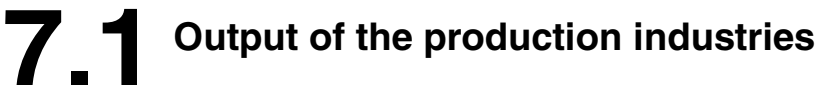

\begin{tabular}{|c|c|c|c|c|c|c|}
\hline & \multicolumn{6}{|c|}{ Market Sector analysis } \\
\hline & \multirow{2}{*}{$\begin{array}{r}\text { Consumer } \\
\text { durables }\end{array}$} & \multirow{2}{*}{$\begin{array}{r}\text { Consumer } \\
\text { non-durables }\end{array}$} & \multirow{2}{*}{$\begin{array}{r}\text { Capital goods } \\
\text { industries }\end{array}$} & \multicolumn{3}{|c|}{ Intermediate goods and energy } \\
\hline & & & & Total & Energy & Intermediate goods \\
\hline \multicolumn{7}{|l|}{ SIC 2003} \\
\hline Weights $^{2}$ & 36.2 & 272.3 & 214.3 & 477.2 & 211.9 & 265.3 \\
\hline & UFIU & UFJS & UFIL & $\mathrm{JMOH}$ & UFJB & UFJL \\
\hline 2004 & 105.0 & 100.5 & 103.6 & 99.6 & 96.5 & 102.2 \\
\hline 2005 & 104.1 & 100.5 & 103.8 & 97.2 & 91.7 & 101.6 \\
\hline 2006 & 106.5 & 101.4 & 107.8 & 96.2 & 87.4 & 103.2 \\
\hline 2007 & 106.3 & 100.0 & 110.5 & 96.7 & 87.1 & 104.2 \\
\hline 2008 & 100.4 & 98.2 & 106.7 & 93.6 & 86.0 & 99.7 \\
\hline \multicolumn{7}{|c|}{ Seasonally adjusted ${ }^{1}$} \\
\hline Q4 & 105.6 & 99.6 & 103.8 & 99.4 & 94.2 & 103.5 \\
\hline 2005 Q1 & 105.5 & 100.7 & 102.5 & 98.5 & 94.2 & 102.0 \\
\hline Q2 & 103.5 & 101.0 & 104.9 & 97.7 & 93.0 & 101.5 \\
\hline Q3 & 103.6 & 100.5 & 105.1 & 96.4 & 90.3 & 101.3 \\
\hline Q4 & 104.0 & 100.0 & 102.7 & 96.4 & 89.5 & 101.8 \\
\hline 2006 Q1 & 104.8 & 101.2 & 106.0 & 97.5 & 91.3 & 102.4 \\
\hline Q2 & 107.4 & 101.0 & 107.9 & 96.1 & 86.9 & 103.3 \\
\hline Q3 & 106.2 & 101.6 & 108.3 & 96.3 & 86.6 & 104.0 \\
\hline Q4 & 107.6 & 101.6 & 109.0 & 95.1 & 85.0 & 103.3 \\
\hline 2007 Q1 & 105.8 & 99.9 & 109.2 & 95.9 & 86.4 & 103.4 \\
\hline Q2 & 106.3 & 100.3 & 111.3 & 96.4 & 86.7 & 104.2 \\
\hline Q3 & 108.1 & 99.6 & 110.5 & 96.9 & 87.1 & 104.7 \\
\hline Q4 & 104.8 & 100.2 & 111.1 & 97.4 & 88.3 & 104.6 \\
\hline 2008 Q1 & $106.2^{\dagger}$ & $100.3^{\dagger}$ & $110.2^{\dagger}$ & $96.0^{\dagger}$ & $86.5^{\dagger}$ & $103.7^{\dagger}$ \\
\hline Q2 & 103.5 & 99.0 & 109.4 & 95.3 & 86.4 & 102.4 \\
\hline 2007 Jan & 106.0 & 100.8 & 109.6 & 95.9 & 86.9 & 103.0 \\
\hline $\mathrm{Feb}$ & 104.8 & 99.5 & 108.3 & 95.8 & 86.6 & 103.2 \\
\hline Mar & 106.7 & 99.5 & 109.7 & 96.0 & 85.8 & 104.1 \\
\hline Apr & 106.2 & 100.4 & 111.0 & 95.1 & 84.5 & 103.7 \\
\hline May & 104.5 & 100.9 & 111.4 & 97.0 & 88.0 & 104.1 \\
\hline Jun & 108.1 & 99.8 & 111.5 & 97.2 & 87.6 & 104.9 \\
\hline Jul & 107.6 & 99.4 & 110.4 & 97.1 & 87.3 & 104.9 \\
\hline Aug & 109.1 & 99.9 & 110.2 & 97.0 & 86.9 & 105.0 \\
\hline Sep & 107.6 & 99.6 & 110.8 & 96.7 & 87.2 & 104.3 \\
\hline Oct & 105.1 & 100.2 & 111.7 & 97.9 & 89.0 & 105.0 \\
\hline Nov & 103.7 & 100.3 & 110.5 & 97.3 & 87.7 & 104.9 \\
\hline Dec & 105.8 & 100.0 & 111.0 & 97.0 & 88.3 & 103.9 \\
\hline 2008 Jan & $105.7^{\dagger}$ & 100.3 & $110.5^{\dagger}$ & $96.1^{\dagger}$ & $86.4^{\dagger}$ & $103.9^{\dagger}$ \\
\hline Feb & 107.4 & $100.5^{\dagger}$ & 110.1 & 96.1 & 86.2 & 104.0 \\
\hline Mar & 105.5 & 100.1 & 109.9 & 95.8 & 86.7 & 103.1 \\
\hline Apr & 104.8 & 99.6 & 110.4 & 95.9 & 87.4 & 102.8 \\
\hline May & 103.1 & 98.9 & 109.5 & 94.9 & 85.4 & 102.5 \\
\hline Jun & 102.5 & 98.4 & 108.1 & 95.1 & 86.4 & 102.1 \\
\hline Jul & 100.1 & 98.7 & 107.9 & 94.2 & 85.4 & 101.3 \\
\hline Aug & 99.2 & 98.4 & 106.6 & 93.4 & 84.7 & 100.3 \\
\hline Sep & 99.6 & 97.3 & 105.3 & 93.8 & 87.2 & 99.1 \\
\hline Oct & 95.4 & 97.2 & 103.9 & 91.1 & 85.0 & 96.0 \\
\hline Nov & 93.3 & 95.1 & 100.2 & 89.2 & 85.1 & 92.4 \\
\hline Dec & 88.2 & 94.3 & 97.7 & 87.7 & 85.8 & 89.3 \\
\hline
\end{tabular}

Note: The figures contain, where appropriate, an adjustment for stock 2 These sum to the total of 1000 for the production industries. changes.

3 Includes adjustments to standardise the length of months.

1 Unadjusted data may be obtained from the Office for National Statistics, IOP

Source: Office for National Statistics: 01633456492 Branch, Government Buildings, Cardiff Road, Newport, NP10 8XG. 


\subsection{Productivity jobs and output per filled job ${ }^{1}$}

$2003=100$

\begin{tabular}{|c|c|c|c|c|c|c|c|c|c|c|c|c|}
\hline & \multirow[b]{2}{*}{$\begin{array}{r}\text { Whole } \\
\text { economy }\end{array}$} & \multirow[b]{2}{*}{$\begin{array}{r}\text { Total } \\
\text { production } \\
\text { industries }\end{array}$} & \multirow[b]{2}{*}{$\begin{array}{r}\text { Total } \\
\text { mining } \\
\text { quarrying } \\
\text { electricity } \\
\text { gas \& water } \\
\text { supply }\end{array}$} & \multicolumn{9}{|c|}{ Manufacturing industries } \\
\hline & & & & $\begin{array}{r}\text { Total } \\
\text { manufac- } \\
\text { turing } \\
\text { indus- } \\
\text { tries }\end{array}$ & $\begin{array}{l}\text { Food, drink } \\
\text { and tobacco }\end{array}$ & $\begin{array}{r}\text { Textiles, } \\
\text { footwear, } \\
\text { clothing } \\
\text { and } \\
\text { leather }\end{array}$ & \multicolumn{2}{|c|}{$\begin{array}{l}\text { Pulp, paper } \\
\text { \& paper } \\
\text { products, Chemicals } \\
\text { printing and } \\
\text { and man-made }\end{array}$} & $\begin{array}{l}\text { Other } \\
\text { non- } \\
\text { metalic } \\
\text { mineral } \\
\text { products }\end{array}$ & $\begin{array}{r}\text { Basic } \\
\text { metals } \\
\text { and fabri- } \\
\text { cated metal a } \\
\text { products }\end{array}$ & $\begin{array}{r}\text { Engi- } \\
\text { neering } \\
\text { and related } \\
\text { industries }\end{array}$ & $\begin{array}{r}\text { Other } \\
\text { manufactur- } \\
\text { ing }\end{array}$ \\
\hline $\begin{array}{l}\text { SIC } 1992 \\
\text { Sub-section }\end{array}$ & & Sect $C+D+E$ & Sect $C+E$ & Sect D & DA & DB_DC & $\mathrm{DE}$ & DG & DI & DJ & DK_DM & $\mathrm{DD}+\mathrm{DF}+\mathrm{DH}+\mathrm{DN}$ \\
\hline \multicolumn{13}{|c|}{ Productivity jobs } \\
\hline & LNNM & LNOJ & LOIW & LNOK & LNOL & LOIS & LOIM & LOIN & LZYL & LZYP & LOIT & LOIZ \\
\hline 2002 & 99.2 & 105.2 & 103.6 & 105.3 & 103.2 & 116.7 & 100.2 & 103.9 & 103.1 & 104.8 & 108.3 & 102.8 \\
\hline 2003 & 100.0 & 100.0 & 100.0 & 100.0 & 100.0 & 100.0 & 100.0 & 100.0 & 100.0 & 100.0 & 100.0 & 100.0 \\
\hline 2004 & 100.8 & 95.7 & 93.5 & 95.8 & 99.0 & 88.9 & 95.4 & 96.3 & 99.5 & 95.8 & 94.9 & 96.6 \\
\hline 2005 & 101.8 & 91.6 & 95.4 & 91.4 & 96.7 & 80.6 & 91.1 & 91.2 & 93.5 & 90.0 & 91.7 & 91.0 \\
\hline 2006 & 102.6 & 90.3 & 102.1 & 89.9 & 93.8 & 74.2 & 92.2 & 88.5 & 89.8 & 91.3 & 89.0 & 91.1 \\
\hline 2007 & 103.4 & 88.2 & 109.4 & 87.1 & 91.7 & 67.1 & 88.6 & 84.3 & 90.5 & 88.7 & 87.0 & 88.2 \\
\hline \multicolumn{13}{|c|}{ Seasonally adjusted } \\
\hline 2004 Q4 & 101.1 & 94.6 & 94.1 & 94.6 & 98.2 & 86.8 & 93.7 & 94.5 & 99.5 & 94.6 & 94.2 & 94.6 \\
\hline 2005 Q1 & 101.6 & 93.3 & 93.4 & 93.3 & 97.7 & 85.7 & 91.7 & 93.1 & 98.0 & 93.0 & 93.7 & 92.1 \\
\hline Q2 & 101.7 & 91.9 & 94.2 & 91.8 & 96.6 & 82.7 & 91.6 & 92.3 & 94.8 & 89.8 & 92.1 & 91.2 \\
\hline Q3 & 102.0 & 90.9 & 96.2 & 90.5 & 96.3 & 78.5 & 90.6 & 90.5 & 92.1 & 88.3 & 91.0 & 90.5 \\
\hline Q4 & 101.9 & 90.3 & 97.6 & 89.8 & 96.2 & 75.6 & 90.3 & 88.8 & 89.1 & 88.9 & 90.0 & 90.3 \\
\hline 2006 Q1 & 102.2 & 89.8 & 97.2 & 89.9 & 95.3 & 75.4 & 91.1 & 88.1 & 89.0 & 90.8 & 89.6 & 90.2 \\
\hline Q2 & 102.5 & 90.3 & 100.2 & 90.0 & 93.8 & 74.4 & 92.3 & 88.5 & 89.9 & 91.6 & 89.3 & 91.0 \\
\hline Q3 & 102.8 & 90.9 & 104.7 & 90.2 & 93.1 & 73.8 & 92.9 & 88.8 & 90.4 & 92.4 & 89.1 & 91.7 \\
\hline Q4 & 102.9 & 90.3 & 106.4 & 89.4 & 93.1 & 73.3 & 92.4 & 88.5 & 90.0 & 90.6 & 88.1 & 91.5 \\
\hline 2007 Q1 & 102.9 & 89.2 & 107.5 & 88.2 & 92.4 & 70.5 & 91.4 & 87.3 & 89.1 & 88.5 & 87.5 & 89.8 \\
\hline Q2 & 103.2 & 88.3 & 108.9 & 87.3 & 91.6 & 67.2 & 89.3 & 85.3 & 89.5 & 88.2 & 87.2 & 88.2 \\
\hline Q3 & 103.6 & 87.9 & 110.1 & 86.6 & 91.2 & 66.2 & 87.6 & 82.6 & 91.8 & 89.6 & 86.9 & 87.1 \\
\hline Q4 & 104.0 & 87.4 & 111.0 & 86.2 & 91.4 & 64.5 & 86.1 & 82.0 & 91.6 & 88.4 & 86.2 & 87.9 \\
\hline 2008 Q1 & 104.4 & 87.0 & 112.9 & 85.6 & 91.3 & 62.4 & 84.1 & 83.4 & 93.0 & 87.7 & 86.0 & 87.0 \\
\hline Q2 & 104.6 & 86.6 & 114.3 & 85.2 & 90.5 & 61.0 & 83.5 & 82.5 & 93.8 & 88.5 & 85.6 & 85.7 \\
\hline Q3 & 104.3 & 85.8 & 115.4 & 84.2 & 89.9 & 60.5 & 83.5 & 81.0 & 90.1 & 86.7 & 85.5 & 83.5 \\
\hline \multicolumn{13}{|c|}{ Output per filled job } \\
\hline & LNNN & LNNW & LOJA & LNNX & LNNY & LNOG & LNOA & LNOB & LZYM & LZYQ & $\mathrm{LNOH}$ & LOJD \\
\hline 2002 & 98.1 & 95.7 & 98.8 & 95.2 & 98.5 & 86.4 & 101.6 & 95.4 & 92.8 & 96.7 & 91.7 & 97.9 \\
\hline 2003 & 100.0 & 100.0 & 100.0 & 100.0 & 100.0 & 100.0 & 100.0 & 100.0 & 100.0 & 100.0 & 100.0 & 100.0 \\
\hline 2004 & 101.9 & 105.4 & 102.4 & 106.6 & 103.0 & 100.3 & 103.7 & 107.6 & 106.6 & 107.2 & 109.9 & 104.7 \\
\hline 2005 & 103.0 & 109.0 & 95.5 & 111.6 & 107.2 & 108.8 & 104.3 & 116.9 & 113.8 & 115.1 & 113.1 & 110.6 \\
\hline 2006 & 105.2 & 111.2 & 85.5 & 115.5 & 109.6 & 118.2 & 102.3 & 124.2 & 121.4 & 115.0 & 121.3 & 111.5 \\
\hline 2007 & 107.5 & 114.3 & 79.4 & 119.9 & 111.6 & 128.5 & 106.0 & 129.1 & 120.9 & 119.9 & 126.1 & 117.2 \\
\hline \multicolumn{13}{|c|}{ Seasonally adjusted } \\
\hline 2004 Q4 & 102.5 & 106.4 & 99.4 & 108.2 & 104.4 & 99.4 & 103.0 & 110.4 & 108.1 & 110.4 & 111.4 & 107.6 \\
\hline 2005 Q1 & 102.4 & 107.4 & 99.2 & 109.3 & 106.4 & 103.2 & 105.3 & 112.3 & 108.0 & 110.0 & 110.1 & 112.4 \\
\hline Q2 & 102.9 & 109.2 & 98.1 & 111.5 & 107.5 & 107.4 & 105.1 & 115.2 & 110.3 & 115.4 & 113.6 & 109.8 \\
\hline Q3 & 103.1 & 109.6 & 93.2 & 112.8 & 107.7 & 110.6 & 104.4 & 118.7 & 115.0 & 116.9 & 115.0 & 110.2 \\
\hline Q4 & 103.8 & 109.6 & 91.6 & 112.9 & 107.2 & 114.0 & 102.5 & 121.3 & 121.7 & 118.0 & 113.9 & 110.0 \\
\hline 2006 Q1 & 104.6 & 112.0 & 93.9 & 114.4 & 107.0 & 118.4 & 104.6 & 122.4 & 123.8 & 114.7 & 118.5 & 111.5 \\
\hline Q2 & 105.1 & 111.1 & 86.4 & 115.4 & 109.3 & 117.6 & 101.2 & 124.1 & 119.1 & 114.8 & 121.2 & 112.7 \\
\hline Q3 & 105.2 & 110.7 & 82.4 & 115.6 & 110.5 & 116.9 & 101.6 & 125.3 & 120.9 & 114.5 & 121.7 & 111.5 \\
\hline Q4 & 105.9 & 111.0 & 79.4 & 116.6 & 111.7 & 120.1 & 101.7 & 125.1 & 121.9 & 116.2 & 123.6 & 110.5 \\
\hline 2007 Q1 & 106.8 & 112.2 & 80.1 & 117.7 & 111.1 & 119.6 & 101.8 & 125.3 & 120.2 & 119.9 & 124.2 & 113.6 \\
\hline Q2 & 107.3 & 114.3 & 79.6 & 119.9 & 112.2 & 128.8 & 105.0 & 127.5 & 124.6 & 121.0 & 126.3 & 116.7 \\
\hline Q3 & 107.9 & 114.8 & 78.9 & 120.6 & 111.9 & 131.9 & 107.0 & 130.6 & 119.9 & 119.1 & 126.4 & 119.5 \\
\hline Q4 & 107.9 & 115.9 & 79.1 & 121.4 & 111.3 & 133.7 & 110.4 & 132.9 & 118.8 & 119.8 & 127.6 & 119.1 \\
\hline 2008 Q1 & 108.0 & 115.7 & 75.1 & 122.4 & 112.0 & 139.2 & 112.6 & 133.3 & 117.8 & 119.6 & 127.8 & 119.3 \\
\hline Q2 & 107.9 & 115.3 & 74.1 & 121.7 & 110.7 & 142.6 & 112.7 & 134.0 & 114.7 & 118.6 & 127.6 & 117.8 \\
\hline Q3 & 107.4 & 114.7 & 72.7 & 121.2 & 111.2 & 144.0 & 110.5 & 137.2 & 114.4 & 119.9 & 124.6 & 118.0 \\
\hline
\end{tabular}

Note: The full productivity and unit wage costs data sets with associated ar-

Source: Office for National Statistics ticles can be found on the National Statistics website at: www.statistics.gov.uk/productivity.

1 Output per filled job is the ratio of the output index numbers published in

Table 7.1 and productivity jobs. A monthly series for total manufacturing industries is presented in Table 7.3.

2 Whole economy output per job is based on Gross Value Added at Basic Prices. 
Production, output and costs

\subsection{Key Productivity Measures}

$2003=100$

\begin{tabular}{|c|c|c|c|c|c|c|}
\hline & \multicolumn{4}{|c|}{ Whole economy } & \multicolumn{2}{|c|}{ Manufacturing industry } \\
\hline & Implied GDP deflator $^{1}$ & $\begin{array}{r}\text { Labour costs per unit } \\
\text { of output }\end{array}$ & $\begin{array}{r}\text { Wages and salaries } \\
\text { per unit of output }\end{array}$ & Output per worker ${ }^{2}$ & $\begin{array}{l}\text { Wages and salaries } \\
\text { per unit of output }\end{array}$ & Output per filled job \\
\hline $\begin{array}{l}2000 \\
2001 \\
2002 \\
2003 \\
2004\end{array}$ & $\begin{array}{r}\text { YBGB } \\
92.2 \\
94.1 \\
97.0 \\
100.0 \\
102.5\end{array}$ & $\begin{array}{r}\text { LNNL } \\
91.7 \\
95.1 \\
97.2 \\
100.0 \\
102.4\end{array}$ & $\begin{array}{r}\text { LNNK } \\
93.2 \\
96.8 \\
98.4 \\
100.0 \\
101.5\end{array}$ & $\begin{array}{r}\text { A4YM } \\
95.8 \\
97.1 \\
98.2 \\
100.0 \\
101.7\end{array}$ & $\begin{array}{r}\text { LNNQ } \\
99.6 \\
100.5 \\
101.4 \\
100.0 \\
97.2\end{array}$ & $\begin{array}{r}\text { LNNX } \\
89.8 \\
92.8 \\
95.2 \\
100.0 \\
106.6\end{array}$ \\
\hline $\begin{array}{l}2005 \\
2006 \\
2007\end{array}$ & $\begin{array}{l}104.8 \\
107.5 \\
110.7\end{array}$ & $\begin{array}{l}105.5 \\
107.7 \\
109.2\end{array}$ & $\begin{array}{l}103.8 \\
105.6 \\
107.5\end{array}$ & $\begin{array}{l}102.8 \\
104.8 \\
107.2\end{array}$ & $\begin{array}{l}96.2 \\
97.8 \\
97.5\end{array}$ & $\begin{array}{l}111.6 \\
115.5 \\
119.9\end{array}$ \\
\hline $\begin{array}{r}2002 \text { Q3 } \\
\text { Q4 }\end{array}$ & $\begin{array}{l}97.4 \\
97.8\end{array}$ & $\begin{array}{l}97.4 \\
98.2\end{array}$ & $\begin{array}{l}98.7 \\
99.0\end{array}$ & $\begin{array}{l}98.5 \\
98.5\end{array}$ & $\begin{array}{l}100.6 \\
101.8\end{array}$ & $\begin{array}{l}96.5 \\
96.2\end{array}$ \\
\hline $\begin{array}{r}2003 \text { Q1 } \\
\text { Q2 } \\
\text { Q3 } \\
\text { Q4 }\end{array}$ & $\begin{array}{r}98.9 \\
99.8 \\
100.4 \\
100.8\end{array}$ & $\begin{array}{r}98.4 \\
99.3 \\
101.0 \\
101.2\end{array}$ & $\begin{array}{r}99.2 \\
99.7 \\
100.7 \\
100.4\end{array}$ & $\begin{array}{r}99.1 \\
99.6 \\
100.2 \\
101.0\end{array}$ & $\begin{array}{r}101.6 \\
100.2 \\
99.8 \\
98.4\end{array}$ & $\begin{array}{r}97.2 \\
99.1 \\
100.6 \\
103.1\end{array}$ \\
\hline $\begin{array}{r}2004 \text { Q1 } \\
\text { Q2 } \\
\text { Q3 } \\
\text { Q4 }\end{array}$ & $\begin{array}{l}101.1 \\
102.2 \\
102.8 \\
103.9\end{array}$ & $\begin{array}{l}101.5 \\
102.0 \\
102.6 \\
103.6\end{array}$ & $\begin{array}{l}100.7 \\
101.1 \\
101.7 \\
102.7\end{array}$ & $\begin{array}{l}100.9 \\
101.9 \\
101.9 \\
102.1\end{array}$ & $\begin{array}{l}96.9 \\
97.2 \\
97.8 \\
96.9\end{array}$ & $\begin{array}{l}105.5 \\
106.6 \\
106.2 \\
108.2\end{array}$ \\
\hline $\begin{array}{r}2005 \text { Q1 } \\
\text { Q2 } \\
\text { Q3 } \\
\text { Q4 }\end{array}$ & $\begin{array}{l}104.2 \\
105.0 \\
104.5 \\
105.5\end{array}$ & $\begin{array}{l}104.3 \\
104.8 \\
105.8 \\
107.0\end{array}$ & $\begin{array}{l}103.6 \\
103.2 \\
103.8 \\
104.8\end{array}$ & $\begin{array}{l}102.1 \\
102.7 \\
102.7 \\
103.5\end{array}$ & $\begin{array}{l}96.9 \\
95.3 \\
95.7 \\
97.0\end{array}$ & $\begin{array}{l}109.3 \\
111.5 \\
112.8 \\
112.9\end{array}$ \\
\hline $\begin{array}{r}2006 \text { Q1 } \\
\text { Q2 } \\
\text { Q3 } \\
\text { Q4 }\end{array}$ & $\begin{array}{l}106.6 \\
106.6 \\
108.2 \\
108.8\end{array}$ & $\begin{array}{l}106.9 \\
107.4 \\
108.2 \\
108.5\end{array}$ & $\begin{array}{l}104.0 \\
106.1 \\
106.4 \\
106.1\end{array}$ & $\begin{array}{l}104.1 \\
104.7 \\
104.8 \\
105.7\end{array}$ & $\begin{array}{l}97.2 \\
97.5 \\
98.3 \\
98.2\end{array}$ & $\begin{array}{l}114.4 \\
115.4 \\
115.6 \\
116.6\end{array}$ \\
\hline $\begin{array}{r}2007 \text { Q1 } \\
\text { Q2 } \\
\text { Q3 } \\
\text { Q4 }\end{array}$ & $\begin{array}{l}109.5 \\
110.4 \\
111.1 \\
111.7\end{array}$ & $\begin{array}{l}109.1 \\
109.2 \\
109.3 \\
109.3\end{array}$ & $\begin{array}{l}107.2 \\
107.1 \\
107.6 \\
108.1\end{array}$ & $\begin{array}{l}106.6 \\
107.0 \\
107.6 \\
107.6\end{array}$ & $\begin{array}{l}97.8 \\
97.3 \\
97.2 \\
97.6\end{array}$ & $\begin{array}{l}117.7 \\
119.9 \\
120.6 \\
121.4\end{array}$ \\
\hline $\begin{array}{r}2008 \text { Q1 } \\
\text { Q2 } \\
\text { Q3 }\end{array}$ & $\begin{array}{l}112.7 \\
113.0 \\
113.4\end{array}$ & $\begin{array}{l}110.8 \\
111.2 \\
112.2\end{array}$ & $\begin{array}{l}108.8 \\
109.5 \\
110.8\end{array}$ & $\begin{array}{l}107.7 \\
107.8 \\
107.4\end{array}$ & $\begin{array}{l}97.6 \\
98.6 \\
99.5\end{array}$ & $\begin{array}{l}122.4 \\
121.7 \\
121.2\end{array}$ \\
\hline $\begin{array}{c}2006 \text { Apr } \\
\text { May } \\
\text { Jun }\end{array}$ & $\begin{array}{l}. . \\
. . \\
. .\end{array}$ & $\begin{array}{l}. . \\
. . \\
. .\end{array}$ & $\begin{array}{l}. . \\
. . \\
. .\end{array}$ & $\begin{array}{l}. . \\
. . \\
. .\end{array}$ & $\begin{array}{l}98.2 \\
96.9 \\
97.3\end{array}$ & $\begin{array}{l}114.6 \\
115.5 \\
116.0\end{array}$ \\
\hline $\begin{array}{l}\text { Jul } \\
\text { Aug } \\
\text { Sep } \\
\text { Oct } \\
\text { Nov } \\
\text { Dec }\end{array}$ & $\begin{array}{l}. . \\
. . \\
. . \\
. \\
. . \\
. .\end{array}$ & $\begin{array}{l}. . \\
. . \\
. . \\
. . \\
. . \\
. .\end{array}$ & $\begin{array}{l}. . \\
. . \\
. . \\
. \\
. . \\
. .\end{array}$ & $\begin{array}{l}. . \\
. . \\
. . \\
. . \\
. . \\
. .\end{array}$ & $\begin{array}{l}97.6 \\
98.6 \\
98.8 \\
98.8 \\
98.0 \\
97.8\end{array}$ & $\begin{array}{l}115.2 \\
115.6 \\
116.0 \\
116.1 \\
116.8 \\
117.0\end{array}$ \\
\hline $\begin{array}{c}2007 \text { Jan } \\
\text { Feb } \\
\text { Mar } \\
\text { Apr } \\
\text { May } \\
\text { Jun }\end{array}$ & $\begin{array}{l}. . \\
. . \\
. . \\
. . \\
. \\
. .\end{array}$ & $\begin{array}{l}. . \\
. . \\
. . \\
. . \\
. . \\
. .\end{array}$ & $\begin{array}{l}. . \\
. . \\
. . \\
. . \\
. . \\
. .\end{array}$ & $\begin{array}{l}. . \\
. . \\
. . \\
. . \\
. . \\
. .\end{array}$ & $\begin{array}{l}97.6 \\
98.5 \\
97.4 \\
97.3 \\
97.4 \\
97.2\end{array}$ & $\begin{array}{l}117.5 \\
117.1 \\
118.3 \\
119.4 \\
119.7 \\
120.6\end{array}$ \\
\hline $\begin{array}{l}\text { Jul } \\
\text { Aug } \\
\text { Sep } \\
\text { Oct } \\
\text { Nov } \\
\text { Dec }\end{array}$ & $\begin{array}{l}. . \\
. . \\
. . \\
. \\
. . \\
. .\end{array}$ & $\begin{array}{l}. . \\
. . \\
. . \\
. . \\
. . \\
. .\end{array}$ & $\begin{array}{l}. . \\
. . \\
. . \\
. \\
. . \\
. .\end{array}$ & $\begin{array}{l}. . \\
. . \\
. . \\
. . \\
. . \\
. .\end{array}$ & $\begin{array}{l}97.3 \\
97.0 \\
97.3^{\dagger} \\
97.0^{\dagger} \\
97.6 \\
98.0\end{array}$ & $\begin{array}{l}120.5 \\
120.8 \\
120.6 \\
121.3 \\
121.5 \\
121.5\end{array}$ \\
\hline $\begin{array}{c}2008 \text { Jan } \\
\text { Feb } \\
\text { Mar } \\
\text { Apr } \\
\text { May } \\
\text { Jun }\end{array}$ & $\begin{array}{l}. . \\
. . \\
. . \\
. . \\
. . \\
. .\end{array}$ & $\begin{array}{l}. . \\
. . \\
. . \\
. . \\
. . \\
. .\end{array}$ & $\begin{array}{l}. . \\
. . \\
. . \\
. . \\
. . \\
. .\end{array}$ & $\begin{array}{l}. . \\
. . \\
. . \\
. . \\
. . \\
. .\end{array}$ & $\begin{array}{l}97.5 \\
97.0 \\
98.3 \\
98.0 \\
98.9 \\
99.0\end{array}$ & $\begin{array}{l}122.1 \\
122.7 \\
122.3 \\
122.1 \\
121.7 \\
121.4\end{array}$ \\
\hline $\begin{array}{l}\text { Jul } \\
\text { Aug } \\
\text { Sep } \\
\text { Oct } \\
\text { Nov }\end{array}$ & $\begin{array}{l}. . \\
. . \\
. . \\
. . \\
. .\end{array}$ & $\begin{array}{l}. . \\
. . \\
. . \\
. . \\
. .\end{array}$ & $\begin{array}{l}. . \\
. . \\
. . \\
. \\
. .\end{array}$ & $\begin{array}{l}. . \\
. . \\
. . \\
. . \\
. .\end{array}$ & $\begin{array}{r}99.2 \\
99.3 \\
100.0 \\
101.0 \\
103.4\end{array}$ & $\begin{array}{l}121.5 \\
121.4 \\
120.8 \\
120.0 \\
117.4\end{array}$ \\
\hline
\end{tabular}

Note: The full productivity and unit wage costs data sets with associated articles can be found on the National Statistics website at: www.statisSource: Office for National Statistics tics.gov.uk/productivity.

1 Based on the sum of expenditure components of GDP at current and constant market prices.

2 Whole Economy output per worker is the ratio of Gross Value Added (GVA) at Basic Prices and Labour Force Survey (LFS) total employment. 